HEALTHY BUILDINGS 



\section{Healthy Buildings}

How Indoor Spaces Drive

Performance and Productivity

JOSEPH G. ALLEN

JOHN D. MACOMBER

II Harvard University Press

Cambridge, Massachusetts

London, England

2020 
Copyright $(9) 2020$ by the President and Fellows of Harvard College All rights reserved

Printed in the United States of America

First printing

Cover artwork: Amin Yusifov / iStock / Getty Images Plus

Cover design: Jill Breitbarth

9780674246089 (EPUB)

9780674246096 (MOBI)

9780674246102 (PDF)

The Library of Congress has cataloged the printed edition as follows:

Names: Allen, Joseph G., 1975- author. | Macomber, John D., author.

Title: Healthy buildings : how indoor spaces drive performance and productivity/Joseph G. Allen, John D. Macomber.

Description: Cambridge, Massachusetts : Harvard University Press, 2020. | Includes index.

Identifiers: LCCN 2019044954 | ISBN 9780674237971 (cloth)

Subjects: LCSH: Industrial productivity—Effect of technological innovations on. | Industrial buildings—-Health aspects. | Sustainable buildings.

Classification: LCC HC79.I52 A55 2020 | DDC 363.11—dc23

LC record available at https://lccn.loc.gov/2019044954 